\title{
ŁOJASIEWICZ INEQUALITY OVER THE RING OF POWER SERIES IN TWO VARIABLES
}

\author{
Guillaume Rond
}

\begin{abstract}
We prove a Łojasiewicz type inequality for a system of polynomial equations with coefficients in the ring of formal power series in two variables. This result is an effective version of the Strong Artin Approximation Theorem. From this result, we deduce a bound of Artin functions of isolated singularities.
\end{abstract}

\section{Introduction}

Let $(A, \mathfrak{m})$ be a Noetherian complete local ring. The powers of the maximal ideal of $A$ define a metric topology

on $A$, called the Krull topology, the norm being defined as

$$
\|z\|:=e^{-\operatorname{ord}(z)}, \quad \forall z \in A
$$

where $\operatorname{ord}(z):=\sup \left\{n \in \mathbb{N} / z \in \mathfrak{m}^{n}\right\}$ for all $z \in A, z \neq 0$. This norm extends to $A^{m}$ using the max norm.

In this paper, we are interested in inequalities relating the distance to the zero set of a polynomial map defined over $A$ to the values of this map. In the case $A$ is a discrete valuation ring (thus a ring of dimension 1) we have the following result:

Theorem 1.1 ([Gr66]). Let $A$ be a complete discrete valuation ring. Let

$$
f(z):=\left(f_{1}(z), \ldots, f_{n}(z)\right) \in A[z]^{n}, \quad z:=\left(z_{1}, \ldots, z_{m}\right) .
$$

Then there exist $a, b \geq 0$ such that

$$
\begin{gathered}
\forall c \in \mathbb{N} \forall \bar{z} \in A^{m} \text { such that } f(\bar{z}) \in \mathfrak{m}^{a c+b} \\
\exists \widetilde{z} \in A^{m} \text { such that } f(\widetilde{z})=0 \text { and } \widetilde{z}_{j}-\bar{z}_{j} \in \mathfrak{m}^{c}, \quad 1 \leq j \leq m .
\end{gathered}
$$

The case $a=0$ in Theorem 1.1 corresponds to the case $f^{-1}(0)=\emptyset$, i.e., there exists a constant $b \in \mathbb{N}$ such that there does not exists $\widetilde{z} \in A^{r}$ with $f(\widetilde{z}) \in \mathfrak{m}^{b}$. In the case $f^{-1}(0) \neq \emptyset$, using the norm defined before it is well known that Theorem 1.1 is equivalent to the following result:

Theorem 1.1'. Let $A$ be a complete discrete valuation ring. Let $f(z) \in A[z]^{n}$ such that $f^{-1}(0) \neq \emptyset$.

$$
\exists \alpha \geq 0, C>0 \text { s.t. }\|f(\bar{z})\| \geq C d\left(f^{-1}(0), \bar{z}\right)^{\alpha} \quad \forall \bar{z} \in A^{m}
$$

where

$$
\|f(\bar{z})\|=\max _{i}\left\|f_{i}(\bar{z})\right\| \text { and } d\left(f^{-1}(0), \bar{z}\right)=\inf _{u \in A / f(u)=0}\|u-\bar{z}\| .
$$

Received by the editors September 5, 2012. 
Proof. Let $\bar{z} \in A^{m}$ and let $c \in \mathbb{N}$ be defined bye $\left.e^{-c}=d\left(f^{-1}(0), \bar{z}\right)\right)$. Then we claim that $f(\bar{z}) \notin \mathfrak{m}^{a(c+1)+b}$. Indeed if it were not the case there would exist $\widetilde{z} \in A^{m}$ such that $f(\widetilde{z})=0$ and $\widetilde{z}_{j}-\bar{z}_{j} \in \mathfrak{m}^{c+1}, 1 \leq j \leq m$, and we would get

$$
d\left(f^{-1}(0), \bar{z}\right) \leq\|\widetilde{z}-\bar{z}\| \leq e^{-(c+1)}<d\left(f^{-1}(0), \bar{z}\right)
$$

which is not possible. Thus $\|f(\bar{z})\|>e^{-(a(c+1)+b)}$, i.e.,

$$
\|f(\bar{z})\| \geq e^{-a-b+1} d\left(f^{-1}(0), \bar{z}\right)^{a} \quad \forall \bar{z} \in A^{m} .
$$

Thus the inequality is satisfied with $C=e^{-a-b+1}$ and $\alpha=a$.

On the other hand, let us assume that Theorem 1.1' is satisfied. Let $\bar{z} \in A^{m}$ be such that $f(\bar{z}) \in \mathfrak{m}^{\alpha c+b}$ where $e^{-b}=C$. Then $\|f(\bar{z})\| \leq C e^{-\alpha c}$. Thus, $d\left(f^{-1}(0), \bar{z}\right) \leq e^{-c}$ and there exists $\widetilde{z} \in A^{m}$ such that $f(\widetilde{z})=0$ and $\widetilde{z}_{j}-\bar{z}_{j} \in \mathfrak{m}^{c}, 1 \leq j \leq m$. This proves that Theorem 1.1 is satisfied.

This kind of inequality is true if we replace $f$ by a real analytic function on an open subset $\Omega$ of $\mathbb{R}^{n}$ and $A$ by a compact $K \subset \Omega$ ([Ł59], see [Te12] for an introduction). This kind of inequality is called a Eojasiewicz inequality. We are interested to extend this Eojasiewicz inequality to the case $A$ is a two-dimensional local complete ring or excellent Henselian local ring. We have the following analogue of Theorem 1.1:

Theorem 1.2 ([Ar69, Po86]). Let $A$ be a complete local ring whose maximal ideal is denoted by $\mathfrak{m}$ and let $f(z):=\left(f_{1}(z), \ldots, f_{n}(z)\right) \in A[z]^{n}$. Then there exists a function $\beta: \mathbb{N} \longrightarrow \mathbb{N}$ such that:

$$
\begin{gathered}
\forall c \in \mathbb{N}, \forall \bar{z}_{1}, \ldots, \bar{z}_{m} \in A \text { s.t. } f(\bar{z}) \in \mathfrak{m}^{\beta(c)} \\
\exists \widetilde{z}_{1}, \ldots, \widetilde{z}_{m} \in A \text { s.t. } f(\widetilde{z})=0 \text { and } \widetilde{z}_{i}-\bar{z}_{i} \in \mathfrak{m}^{c} 1 \leq i \leq m .
\end{gathered}
$$

This theorem has been been proved by M. Artin in the case $A$ is the henselization of the ring of polynomials over a discrete valuation ring and by Popescu in the general case.

Definition 1.3. The least function $\beta$ satisfying Theorem 1.2 is called the Artin function of $f$. This is an increasing function that depends only on the ideal $I:=$ $\left(f_{1}(z), \ldots, f_{n}(z)\right)$. See $[\mathrm{Ro} 06]$ for properties of this function.

M. Artin raised the problem of finding estimates on the growth of Artin functions [Ar70]. In general they are not bounded by affine functions as in Theorem 1.1 (in [Ro05] it is shown that the Artin function of $z_{1}^{2}-z_{2}^{2} z_{3}$ is not bounded by an affine function if $A=\mathbb{k} \llbracket x, y \rrbracket)$, thus there is no Łojasiewicz inequality as in Theorem 1.1' in this context. However, Artin's question remains widely open in general. As Theorem 1.1 is equivalent to Theorem 1.1', Theorem 1.2 is equivalent to the following result:

Theorem 1.2'. Let $A$ be a complete local ring and let $f(z) \in A[z]^{n}$ such that $f^{-1}(0) \neq$

$\emptyset$. Then there exists a increasing continuous function $\gamma: \mathbb{R}_{\geq 0} \longrightarrow \mathbb{R}_{\geq 0}$ such that $\gamma(0)=0$ and

$$
\|f(\bar{z})\| \geq \gamma\left(d\left(f^{-1}(0), \bar{z}\right)\right) \quad \forall \bar{z} \in A^{m}
$$


Proof. The proof is exactly the same as the proof that Theorem 1.1 is equivalent to Theorem 1.1'. We have to replace $a c+b$ by $\beta(c)$ and $\gamma(t):=\exp (-\beta(-\ln t+1))$ for any $t \in \ln ^{-1}(\mathbb{N})$. Since $\beta$ may be chosen to be an increasing function, $\gamma$ is increasing and may be continuously extended to a continuous function on $\mathbb{R}_{\geq 0}$. Moreover, saying that $\gamma(0)=0$ is equivalent to say that $\beta(c)$ goes to infinity as $c$ goes to infinity.

The aim of this paper is to give an analogue to Łojasiewicz inequality when $A=$ $\mathbb{k} \llbracket x, y \rrbracket$ and $\mathbb{k}$ is a perfect field (see Theorem 4.4). It asserts that the Artin function of $I$ is bounded by a linear function if the approximated solutions are not too close to the singular locus of $I$. This is a generalization of the main result of [Ro10], where a similar result is proven for binomials ideals. The proof is inspired by the proof of M. Artin of Theorem 1.2 (see [Ar69]): we use the Weierstrass division theorem in order to divide $f$ by a well-chosen minor of the Jacobian matrix of $f$ helping us to reduce the problem to the case of a system of equations with coefficients in $\mathbb{k} \llbracket x \rrbracket$. Then we use an effective version of Theorem 1.1 proven in [Ro10]. Finally, we deduce from Theorem 4.4 that the Artin function of an isolated singularity is bounded by a doubly exponential function (see Corollary 4.13).

\section{Notations}

Let $(A, \mathfrak{m})$ be a local ring. Let us denote by ord the $\mathfrak{m}$-adic order on $A$, i.e., $\operatorname{ord}(z):=$ $\sup \left\{n \in \mathbb{N} / z \in \mathfrak{m}^{n}\right\}$, where $\mathfrak{m}$ is the maximal ideal of $A$. This order function defines a norm on $A$ as follows:

$$
\|z\|:=e^{-\operatorname{ord}(z)}, \quad \forall z \in A .
$$

This is an ultrametric norm, i.e., $\left\|z+z^{\prime}\right\| \leq \max \left\{\|z\|,\left\|z^{\prime}\right\|\right\}$ since $\operatorname{ord}\left(z+z^{\prime}\right) \geq$ $\min \left\{\operatorname{ord}(z), \operatorname{ord}\left(z^{\prime}\right)\right\}$ for any $z, z^{\prime} \in A$. Since $\operatorname{ord}\left(z z^{\prime}\right) \geq \operatorname{ord}(z)+\operatorname{ord}\left(z^{\prime}\right)$, we have $\left\|z z^{\prime}\right\| \leq\|z\| \cdot\left\|z^{\prime}\right\|$ for any $z, z^{\prime} \in A$. We can extend this norm on $A^{m}$ by taking the maximum of the norms of the coordinates:

$$
\|z\|:=\max _{1 \leq i \leq m}\left\|z_{i}\right\|, \quad \forall z:=\left(z_{1}, \ldots, z_{m}\right) \in A^{m}
$$

This norm defines a metric on $A^{m}$ as follows: $d\left(z, z^{\prime}\right):=\left\|z-z^{\prime}\right\|$ for any $z, z^{\prime} \in A^{m}$.

If $a=\left(a_{1}, \ldots, a_{n}\right) \in A^{n}$ and $c \in \mathbb{N}$, writing $a \in \mathfrak{m}^{c}$ will mean $a_{i} \in \mathfrak{m}^{c}$ for all $1 \leq i \leq n$.

\section{Jacobian ideal}

Definition 3.1 ([El73]). Let $A$ be a Noetherian ring and let $f_{1}, \ldots, f_{n} \in$ $A\left[z_{1}, \ldots, z_{m}\right]$. Let $E$ be a subset of $\llbracket 1, n \rrbracket$ of cardinal $h$. We denote by $\Delta_{E}(f)$ the ideal of $A[z]$ generated by the $h \times h$ minors of the Jacobian matrix $\left(\frac{\partial f_{i}}{\partial z_{j}}\right)_{i \in E, 1 \leq j \leq m}$ (This ideal is zero if $h>m$ ). We define the following ideal of $A[z]$ :

$$
H_{f_{1}, \ldots, f_{n}}:=\sum_{E} \Delta_{E}(f)\left(\left(f_{i}, i \in E\right): I\right)
$$

where the sum runs over all subsets $E$ of $\llbracket 1, n \rrbracket$. 
Remark 3.2. Apparently, this definition depends on the choice of the generators $f_{1}, \ldots, f_{n}$ of $I$ and no details are given in [El73]. In most references using Elkik's definition nothing is said about the dependence of $H_{f_{1}, \ldots, f_{n}}$ on the choice of the generators either it is just said that it is easy to check that it does not depend on this choice.

In fact, a prime ideal of $\frac{A[z]}{I}$ is in the smooth locus of the scheme $\operatorname{Spec}\left(\frac{A[z]}{I}\right)$ if and only if it does not contain $H_{f_{1}, \ldots, f_{n}} \frac{A[z]}{I}$ (see for example Prop. 2.13 [Sp99] or Prop. 5.3 $[\mathrm{Po} 00])$, i.e., the smooth locus of $\operatorname{Spec}\left(\frac{A[z]}{I}\right)$ is $\operatorname{Spec}\left(\frac{A[z]}{I}\right) \backslash V\left(\sqrt{H_{f_{1}, \ldots, f_{n}}} \frac{A[z]}{I}\right)$. In particular $\sqrt{H_{f_{1}, \ldots, f_{n}}} \frac{A[z]}{I}$ does not depend on the presentation of the $A$-algebra $\frac{A[z]}{I}$. Another definition of an ideal containing $H_{f_{1}, \ldots, f_{n}}$ whose support is the non-smooth locus of $\operatorname{Spec}\left(\frac{A[z]}{I}\right)$ and which is independent of the presentation of $\frac{A[z]}{I}$ over $A$ is given in ([G-R03] Definition 5.4.1).

Nevertheless in general the image of $H_{f_{1}, \ldots, f_{n}}$ in $\frac{A[z]}{I}$ depends on the generators $f_{1}, \ldots, f_{n}$ as we can see in the following example:

Example 3.3. Set $A=\mathbb{Q}[x, y, z, t]$,

$$
f_{1}:=x z, f_{2}:=x t, \quad f_{3}:=y z, \quad f_{4}:=y t
$$

and let $I$ be the ideal of $A$ generated by $f_{1}, \ldots, f_{4}: I=(x, y) \cap(z, t)$. The Jacobian matrix of $f_{1}, \ldots, f_{4}$ is

$$
M:=\left(\begin{array}{llll}
\frac{\partial f_{1}}{\partial x} & \frac{\partial f_{2}}{\partial x} & \frac{\partial f_{3}}{\partial x} & \frac{\partial f_{4}}{\partial x} \\
\frac{\partial f_{1}}{\partial y} & \frac{\partial f_{2}}{\partial y} & \frac{\partial f_{3}}{\partial y} & \frac{\partial f_{4}}{\partial y} \\
\frac{\partial f_{1}}{\partial z} & \frac{\partial f_{2}}{\partial z} & \frac{\partial f_{3}}{\partial z} & \frac{\partial f_{4}}{\partial z} \\
\frac{\partial f_{1}}{\partial t} & \frac{\partial f_{2}}{\partial t} & \frac{\partial f_{3}}{\partial t} & \frac{\partial f_{4}}{\partial t}
\end{array}\right)=\left(\begin{array}{cccc}
z & t & 0 & 0 \\
0 & 0 & z & t \\
x & 0 & y & 0 \\
0 & x & 0 & y
\end{array}\right) .
$$

Then $\operatorname{det}(M)=x y z t-x y z t=0$. Let us compute $H_{f_{1}, \ldots, f_{4}}$ modulo $I$ :

All the $3 \times 3$ minors of $M$ are in $I$. Let us compute the $2 \times 2$ minors of $M$ which are not in $I$. The only one involving $f_{1}$ and $f_{2}$ is $x^{2}$, the only one involving $f_{3}$ and $f_{4}$ is $y^{2}$. The only one involving $f_{1}$ and $f_{3}$ is $z^{2}$, the only one involving $f_{2}$ and $f_{4}$ is $t^{2}$. Those involving $f_{1}$ and $f_{4}$ and $f_{2}$ and $f_{3}$ are $x y$ and $z t$.

Now let us compute the ideals $\left(\left(f_{i}, f_{j}\right): I\right)$ modulo $I$ for $1 \leq i<j \leq 4$ :

$$
\begin{aligned}
&\left(\left(f_{1}, f_{2}\right): I\right)=(x) \text { mod. } I \\
&\left(\left(f_{1}, f_{3}\right): I\right)=(z) \bmod . I \\
&\left(\left(f_{1}, f_{4}\right): I\right)=\left(\left(f_{2}, f_{3}\right): I\right)=(x y, z t) \bmod . I \\
&\left(\left(f_{2}, f_{4}\right): I\right)=(t) \bmod . I \\
&\left(\left(f_{3}, f_{4}\right): I\right)=(y) \bmod . I
\end{aligned}
$$

Moreover, the ideals $\left(\left(f_{i}\right): I\right)=0$ modulo $I$ for any $1 \leq i \leq 4$. Thus, we obtain

$$
H_{f_{1}, \ldots, f_{4}}=\left(x^{3}, y^{3}, z^{3}, t^{3},(x y)^{2},(z t)^{2}\right) \text { modulo } I \text {. }
$$


Now let us consider

$$
h_{1}:=x(z+t), h_{2}:=x(z-t), h_{3}:=y z, h_{4}=y t .
$$

These four elements generate $I$. The Jacobian matrix of $h_{1}, \ldots, h_{4}$ is

$$
N:=\left(\begin{array}{llll}
\frac{\partial h_{1}}{\partial x} & \frac{\partial h_{2}}{\partial x} & \frac{\partial h_{3}}{\partial x} & \frac{\partial h_{4}}{\partial x} \\
\frac{\partial h_{1}}{\partial y} & \frac{\partial h_{2}}{\partial y} & \frac{\partial h_{3}}{\partial y} & \frac{\partial h_{4}}{\partial y} \\
\frac{\partial h_{1}}{\partial z} & \frac{\partial h_{2}}{\partial z} & \frac{\partial h_{3}}{\partial z} & \frac{\partial h_{4}}{\partial z} \\
\frac{\partial h_{1}}{\partial t} & \frac{\partial h_{2}}{\partial t} & \frac{\partial h_{3}}{\partial t} & \frac{\partial h_{4}}{\partial t}
\end{array}\right)=\left(\begin{array}{cccc}
z+t & z-t & 0 & 0 \\
0 & 0 & z & t \\
x & x & y & 0 \\
x & -x & 0 & y
\end{array}\right) .
$$

Let us now compute $H_{h_{1}, \ldots, h_{4}}$ modulo $I$ :

As before $\operatorname{det}(N)=0$ and all the $3 \times 3$ minors of $N$ are in $I$. Let us compute the $2 \times 2$ minors of $N$, which are not in $I$. The only one involving $h_{1}$ and $h_{2}$ is $x^{2}$. The only one involving $h_{3}$ and $h_{4}$ is $y^{2}$. The only ones involving $h_{1}$ and $h_{3}$ are $x y$ and $z(z+t)$. Those involving $h_{2}$ and $h_{4}$ are $x y$ and $t(z-t)$. Those involving $h_{1}$ and $h_{4}$ are $x y$ and $t(z+t)$ and those involving $h_{2}$ and $h_{3}$ are $x y$ and $z(z-t)$.

Now let us compute the ideals $\left(\left(h_{i}, h_{j}\right): I\right)$ modulo $I$ for $1 \leq i<j \leq 4$ :

$$
\begin{gathered}
\left(\left(h_{1}, h_{2}\right): I\right)=(x) \text { mod. } I \\
\left(\left(h_{1}, h_{3}\right): I\right)=(x y, z(z+t)) \bmod . I \\
\left(\left(h_{1}, h_{4}\right): I\right)=(x y, t(z+t)) \bmod . I \\
\left(\left(h_{2}, h_{3}\right): I\right)=(x y, z(z-t)) \text { mod. } I \\
\left(\left(h_{2}, h_{4}\right): I\right)=(x y, t(z-t)) \text { mod. } I \\
\left(\left(h_{3}, h_{4}\right): I\right)=(y) \text { mod. } I
\end{gathered}
$$

Moreover, $\left(\left(h_{i}\right): I\right)=0$ modulo $I$ for any $1 \leq i \leq 4$. Thus, we obtain

$$
H_{h_{1}, \ldots, h_{4}}=\left(x^{3}, y^{3},(x y)^{2}, z^{2}(z+t)^{2}, t^{2}(z+t)^{2}, z^{2}(z-t)^{2}, t^{2}(z-t)^{2}\right) \text { modulo } I \text {. }
$$

Clearly $H_{h_{1}, \ldots, h_{4}} \subset H_{f_{1}, \ldots, f_{4}}$ modulo $I$. On the other hand, $z^{3} \in H_{f_{1}, \ldots, f_{4}}+I$. If $z^{3} \in H_{h_{1}, \ldots, h_{4}}+I$ then $z^{3} \in H_{h_{1}, \ldots, h_{4}}+I$ modulo $(x, y, t)$. But

$$
H_{h_{1}, \ldots, h_{4}}+I=\left(z^{4}\right) \bmod .(x, y, t)
$$

and $z^{3} \notin\left(z^{4}\right)$. Thus $z^{3} \notin H_{h_{1}, \ldots, h_{4}}+I$ and $H_{f_{1}, \ldots, f_{4}} \neq H_{h_{1}, \ldots, h_{4}}$ modulo $I$.

In fact, we can show more: let us denote by $\bar{J}$ the integral closure of an ideal $J$. Since $H_{h_{1}, \ldots, h_{4}}+I \subset H_{f_{1}, \ldots, f_{4}}+I$, we have $\overline{H_{h_{1}, \ldots, h_{4}}+I} \subset \overline{H_{f_{1}, \ldots, f_{4}}+I}$. However, since $\overline{\left(z^{k}\right)}=\left(z^{k}\right)$ for any integer $k$, we see that

$$
\overline{H_{f_{1}, \ldots, f_{4}}+I} \subsetneq \overline{H_{h_{1}, \ldots, h_{4}}+I} .
$$

We finish this section by giving some effective bounds on $H_{f_{1}, \ldots, f_{n}}$ that we need in the proof of Theorem 4.4.

Lemma 3.4. Let $\mathbb{k}$ be a field. Let $I$ be an ideal of $\mathbb{k} \llbracket x, y \rrbracket\left[z_{1}, \ldots, z_{m}\right]$, where $x$ and $y$ are single variables, generated by polynomials $f_{1}, \ldots, f_{n}$ of degree $\leq d$. Then $H_{f_{1}, \ldots, f_{n}}$ is generated by polynomials of degree $\leq(m+2)\left((d+m+2)^{m+2} d\right)^{2^{m+1}}+(m+2)(d-1)$. 
Proof. The ideal $H_{f_{1}, \ldots, f_{n}}$ is generated by the products of one generator of the ideal $\left(\left(f_{i}, i \in E\right): I\right)$ and of one generator of $\Delta_{E}(f)$. If the cardinal of $E$ equals $h$, then $\Delta_{E}(f)$ is generated by polynomials of degree $\leq h(d-1)$. Moreover $\left(\left(f_{i}, i \in E\right): I\right)$ is generated by polynomials of degree $\leq(m+2)\left((d+m+2)^{m+2} d\right)^{2^{m+1}}$ (cf. 56 [Se74]). Since $h \leq m+2$ this proves the lemma.

Corollary 3.5. Let $I$ be an ideal of $\mathbb{k} \llbracket x, y \rrbracket\left[z_{1}, \ldots, z_{m}\right]$ generated by polynomials $f_{1}, \ldots, f_{n}$ of degree $\leq d$. Let $H$ be any ideal of $\mathbb{k} \llbracket x, y \rrbracket[z]$ such that $\sqrt{H+I}=$ $\sqrt{H_{f_{1}, \ldots, f_{n}}+I}$. Then we have

$$
(H+I)^{e} \subset H_{f_{1}, \ldots, f_{n}}+I,
$$

where

$$
e:=\left((m+2)\left((d+m+2)^{m+2} d\right)^{2^{m+1}}+(m+2)(d-1)\right)^{\min \{n, m+1\}} .
$$

Proof. By Théorème 1 [Te90] we have

$$
\sqrt{J}^{d^{\min \{n, m+1\}}} \subset J
$$

for any ideal $J$ of $\mathbb{k} \llbracket x, y \rrbracket\left[z_{1}, \ldots, z_{m}\right]$ generated by $n$-polynomials of degree $\leq d$. We apply this to the ideal $J:=H_{f_{1}, \ldots, f_{n}}+I$ using Lemma 4.3.

Remark 3.6. By Proposition 2.13 [Sp99] or Proposition 5.3 [Po00], we can choose $H:=\sqrt{H_{f_{1}, \ldots, f_{n}}}$ or

$$
H:=\sum_{g} \sum_{E} \Delta_{E}(g)\left(\left(g_{i}, i \in E\right): I\right)
$$

where the first sum runs over all the sets of generators $g_{1}, \ldots, g_{s}$ of $I$ and the second sum runs over all subsets $E$ of $\llbracket 1, s \rrbracket$.

Remark 3.7. We claim that there exists a constant $C>1$ such that for all $d \geq 2$ and all $m \geq 1, e \leq d^{C^{m}}$.

Indeed, for all $d \geq 2$ and $m \geq 1$, we have

$$
\begin{aligned}
e \leq\left(2(m+2)(d+m+2)^{m+2} d\right)^{2^{m+1} m} & \leq(d+m+2)^{2^{m+1} m(m+5)} \\
& \leq(d+m+2)^{2^{3(m+1)}} \leq(d+m+2)^{64^{m}} .
\end{aligned}
$$

But

$$
\log (d+m+2) \leq C^{\prime m} \log (d)
$$

for all $d \geq 2$ and $m \geq 1$ and a well chosen constant $C^{\prime}>0$. Thus we set $C:=64 C^{\prime}$ and the claim is proven.

\section{4. Łojasiewicz inequality with respect to the Krull topology}

Definition 4.1. Let $I$ be an ideal of $A[z]$ and let $\bar{z} \in A^{m}$. We say $I(\bar{z}) \in \mathfrak{m}^{\beta}$ if and only if $g(\bar{z}) \in \mathfrak{m}^{\beta}$ for all $g \in I$.

The set $I^{-1}(0)$ is defined as

$$
I^{-1}(0):=\left\{\bar{z} \in A^{m} / g(\bar{z})=0 \quad \forall g \in I\right\} .
$$

Let us recall the following result that we will used in the proof of Theorem 4.4: 
Theorem 4.2 ([Ro10]). Let $\mathbb{k}$ be a perfect field. For all $m, d \in \mathbb{N}$, there exists $a(m, d) \in \mathbb{Z}$ such that for any $f=\left(f_{1}, \ldots, f_{n}\right) \in \mathbb{k}[x, z]^{n}$, with $z=\left(z_{1}, \ldots, z_{m}\right)$ and $x$ a single variable, such that the total degree of $f_{i}$ is less or equal to $d$ for $1 \leq i \leq n$, for all $c \in \mathbb{N}$ and for all $z(x) \in \mathbb{k}[[x]]^{m}$, such that $f(x, z(x)) \in(x)^{a(m, d)(c+1)}$, there exists $\bar{z}(x) \in \mathbb{k}[[x]]^{m}$ such that $f(x, \bar{z}(x))=0$ and $z(x)-\bar{z}(x) \in(x)^{c}$. Moreover, the function $(m, d) \longmapsto a(m, d)$ is a polynomial function with respect to $d$ whose degree is exponential in $m$.

Remark 4.3. In [Ro10] this theorem is stated for a characteristic zero field $\mathbb{k}$. In fact the hypothesis on the characteristic of the field is made only for using the Jacobian criterion. However, this is one is still valid if $\mathbb{k}$ is perfect. Thus this theorem is valid for any perfect field $\mathbb{k}$.

Then we can state our main theorem:

Theorem 4.4. Let $A:=\mathbb{k} \llbracket x, y \rrbracket, x$ and $y$ being single variables, and $\mathbb{k}$ be a perfect field. Then there exist constants $K_{1}, K_{2}, K_{3}>0$ such that for any $d \geq 2$ and any $m \geq 1$, for any ideal $I=\left(f_{1}, \ldots, f_{n}\right)$ of $\mathbb{k}[x, y, z]$ generated by polynomials of degrees less than $d$ such that $f^{-1}(0) \neq \emptyset$, where $z:=\left(z_{1}, \ldots, z_{m}\right)$, we have the following inequalities:

$$
\begin{aligned}
& \|f(\bar{z})\| \geq\left(K_{1} d\left(\bar{z}, f^{-1}(0)\right)\right)^{d}\left(\frac{1}{\left\|H_{f_{1}, \ldots, f_{n}}(\bar{z})\right\|}\right)^{K_{2} m} \quad \forall \bar{z} \in A^{m} \backslash H_{f_{1}, \ldots, f_{n}}^{-1}(0) \\
& \|f(\bar{z})\| \geq\left(K_{1} d\left(\bar{z}, f^{-1}(0)\right)\right)^{d}\left(\frac{1}{\|H(\bar{z})\|}\right)^{d^{K_{3}^{m}}} \quad \forall \bar{z} \in A^{m} \backslash H^{-1}(0)
\end{aligned}
$$

where $H$ is any ideal of $A[z]$ such that $\sqrt{H+I}=\sqrt{H_{f_{1}, \ldots, f_{n}}+I}$.

Remark 4.5. Both inequalities show that we have a Łojasiewicz inequality as in Theorem 1.1' if we consider elements $\bar{z}$, whose contact order with the singular locus of $X:=\operatorname{Spec}\left(\frac{A[z]}{I}\right)$ i.e. $\operatorname{ord}(H(\bar{z}))$, where $H$ is an ideal defining the singular locus of $X$, is bounded.

Remark 4.6. In Theorem 4.4, both inequalities are valid only when $\bar{z} \notin H^{-1}(0)$. In general we can do the following:

Let $e$ be an integer such that $\sqrt{I}^{e} \subset I$. Let $f_{1}, \ldots, f_{n}$ be generators of $I$ and $g_{1}, \ldots, g_{l}$ be generators of $\sqrt{I}$. For any $\bar{z} \in A^{n}$ and for any $i$ we have $g_{i}^{e}(\bar{z}) \in$ $\left(f_{1}(\bar{z}), \ldots, f_{n}(\bar{z})\right)$. Thus for any $i$, there exist $a_{i, 1}, \ldots, a_{i, n} \in A$ such that

$$
g_{i}^{e}(\bar{z})=a_{i, 1} f_{1}(\bar{z})+\cdots+a_{i, n} f_{n}(\bar{z}) .
$$

Hence $\operatorname{ord}\left(g_{i}^{e}(\bar{z})\right) \geq \min _{j} \operatorname{ord}\left(f_{j}(\bar{z})\right)$ and $\|g(\bar{z})\|^{e} \leq\|f(\bar{z})\|$. Since $f^{-1}(0)=g^{-1}(0)$ we see that if $g_{1}, \ldots, g_{l}$ satisfy Inequality (4.1) or $(4.2)$ then $f_{1}, \ldots, f_{n}$ satisfy the same kind of inequality where $d\left(f^{-1}(0), \bar{z}\right)$ is replaced by $d\left(f^{-1}(0), \bar{z}\right)^{e}$.

In particular if $I$ is a radical ideal and if we set $X:=\operatorname{Spec}\left(\frac{A}{I}\right)$, then $H$ defines the singular locus of $X$ denoted by $\operatorname{Sing}(X)$ which is a proper closed subset of $X$. Then we have a natural stratification of $X$ where the first stratum is $\operatorname{Reg}(X)$, the regular locus of $X$, the second one is $\operatorname{Reg}(\operatorname{Sing}(X))$, the third one is $\operatorname{Reg}(\operatorname{Sing}(\operatorname{Sing}(X)))$, etc. On each of these strata, we can apply Theorem 4.4. Thus, we see that we can stratify 
$X$ into a finite set of locally closed subsets of $X$, such that on each stratum $S$ we have an inequality of the form

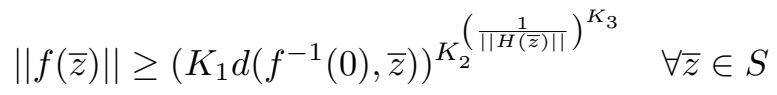

where $\bar{S}=f^{-1}(0)(\bar{S}$ denotes the Zariski closure of $S)$ and $S=\bar{S} \backslash H^{-1}(0)$.

By replacing $H$ by some power of $H$, we may even assume that $K_{3}=1$.

Remark 4.7. If $f_{1}, \ldots, f_{n}$ are polynomials of degree 1 with respect to $z$, then the Artin function of $f$ is bounded by an affine function (cf. Théorème 3.1 [Ro06]). Thus such a system satisfies Theorem 1.1'.

Remark 4.8. This theorem is not true if $A$ is of dimension more than 2. In [Ro06] the following example is given:

Let $A:=\mathbb{k} \llbracket x_{1}, x_{2}, x_{3} \rrbracket$ and let $f:=z_{1} z_{2}-z_{3} z_{4}$. Here $H_{f}=\sqrt{H_{f}}=\left(z_{1}, z_{2}, z_{3}, z_{4}\right)$. For any $c \geq 3$, let us denote

$$
\bar{z}_{1}^{(c)}:=x_{1}^{c}, \quad \bar{z}_{2}^{(c)}:=x_{2}^{c}, \quad \bar{z}_{3}^{(c)}:=x_{1} x_{2}-x_{3}^{c} .
$$

Then there exists $\bar{z}_{4}^{(c)} \in A$ such that $\bar{z}_{1}^{(c)} \bar{z}_{2}^{(c)}-\bar{z}_{3}^{(c)} \bar{z}_{4}^{(c)} \in \mathfrak{m}^{c^{2}}$. Moreover it is proved in [Ro06], that any solution $\widetilde{z} \in A^{4}$ of $f=0$ satisfies $\min _{i=1, \ldots, 4}\left\{\operatorname{ord}\left(\bar{z}_{i}^{(c)}-\widetilde{z}_{i}\right)\right\} \leq c$. Thus there do not exist constants $a>0$ and $b>0$ such that $\left\|f\left(\bar{z}^{(c)}\right)\right\| \geq \operatorname{ad}\left(\bar{z}^{(c)}, f^{-1}(0)\right)^{b}$ for all $c \in \mathbb{N}$, but $\left\|H_{f}\left(\bar{z}^{(c)}\right)\right\|=e^{-2}$ is constant for any $c$.

Remark 4.9. This theorem is still valid if $A$ is any excellent Henselian local ring whose completion is $\mathbb{k} \llbracket x, y \rrbracket$ by Artin Approximation Theorem [Po86]. Indeed in this case the zero set of $f$ in $A$ is dense in the zero set of $f$ in $\mathbb{k} \llbracket x, y \rrbracket$ for the topology induced by the norm $\|$.$\| .$

Proof of Theorem 4.4. We begin to prove the first inequality. Let us denote by $\mathfrak{m}:=$ $(x, y)$ the maximal ideal of $\mathbb{k} \llbracket x, y \rrbracket$. Let $c \in \mathbb{N}$. Let $s \in \mathbb{N}$ and let $\bar{z} \in \mathbb{k} \llbracket x, y \rrbracket^{m}$ such that $f(\bar{z}) \in \mathfrak{m}^{\gamma}$ for all $f \in I$ with

$$
\gamma=\gamma(m, d, s, c):=a(2(m+1) s, 4 m d s)(c+2 s+1)
$$

where $a(.,$.$) is the function of Theorem 4.2$ and let us assume that $H_{f_{1}, \ldots, f_{n}}(\bar{z}) \not \subset \mathfrak{m}^{s}$. Since $H_{f_{1}, \ldots, f_{n}}$ is generated by the elements $\delta_{E} k_{E}$ where $\delta_{E}$ is a minor of the Jacobian matrix $\left(\frac{\partial f_{i}}{\partial z_{j}}\right)_{i \in E, 1 \leq j \leq m}$ and $\left.k_{E} \in\left(f_{i}, i \in E\right): I\right)$, there exists $E \subset \llbracket 1, n \rrbracket$ such that $\delta_{E}(\bar{z}) k_{E}(\bar{z}) \notin \mathfrak{m}^{s}$. In particular $\delta_{E}(\bar{z}) \notin \mathfrak{m}^{s}$. Let $\delta$ denote this minor, i.e., $\delta:=\delta_{E}$. Then we remark that $\operatorname{deg}(\delta) \leq m(d-1)$.

For convenience we will assume that $E=\{1, \ldots, q\}$ where $q \leq n$.

Let $r:=\operatorname{ord}\left(\delta^{2}(\bar{z})\right) \leq 2(s-1)$. In this case ord $\left(k_{E}(\bar{z})\right)<s-\frac{\bar{r}}{2}$. If $r=0$ then $\delta^{2}(\bar{z})$ is invertible and $f(\bar{z}) \in\left(\delta^{2}(\bar{z})\right) \mathfrak{m}^{\gamma} \subset\left(\delta^{2}(\bar{z})\right) \mathfrak{m}^{c}$. In this case we set $\overline{\bar{z}}:=\bar{z}$. Then let us assume that $\delta^{2}(\bar{z})$ is not invertible. Since $\mathbb{k}$ is infinite, by making a linear change of variables in $x$ and $y$, we may assume that $\delta^{2}(\bar{z})$ is regular with respect to $y$ and by the Weierstrass Preparation Theorem $\delta^{2}(\bar{z})=\bar{u} \bar{a}$ where $\bar{u}$ is a unit and

$$
\bar{a}:=y^{r}+\bar{a}_{1}(x) y^{r-1}+\cdots+\bar{a}_{r}(x),
$$

where $a_{i}(x) \in(x)^{r-i} \mathbb{k} \llbracket x \rrbracket, 1 \leq i \leq r$. 
Then we perform the Weierstrass division of $\bar{z}_{i}$ by $\bar{a}$ :

$$
\bar{z}_{i}=\bar{a} \bar{w}_{i}+\sum_{j=0}^{r-1} \bar{z}_{i, j}(x) y^{j}
$$

for $1 \leq i \leq m$. Set

$$
\bar{z}_{i}^{*}:=\sum_{j=0}^{r-1} \bar{z}_{i, j}(x) y^{j}, \quad 1 \leq i \leq m .
$$

Then $\delta^{2}(\bar{z})=\delta^{2}\left(\bar{z}^{*}\right) \bmod . \bar{a}$ and $f_{k}(\bar{z})=f_{k}\left(\bar{z}^{*}\right) \bmod . \bar{a}$ for $1 \leq k \leq n$.

Let $z_{i, j}, 1 \leq i \leq m, 0 \leq j \leq r-1$, be new variables. Let us define $z_{i}^{*}:=\sum_{j=0}^{r-1} z_{i, j} y^{j}$, $1 \leq i \leq m$, and

$$
A\left(a_{i}, y\right):=y^{r}+a_{1} y^{r-1}+\cdots+a_{r} \in \mathbb{k}\left[y, a_{1}, \ldots, a_{r}\right],
$$

where $a_{1}, \ldots, a_{r}$ are new variables. Then the Euclidean division of $\delta^{2}\left(z^{*}\right)$ and $f_{i}\left(z^{*}\right)$ by $A$ (seen as a polynomial in $y$ ) may be written as follows:

$$
\begin{gathered}
\delta^{2}\left(z^{*}\right)=A \cdot Q+\sum_{l=0}^{r-1} G_{l} y^{l} \\
f_{k}\left(z^{*}\right)=A \cdot Q_{k}+\sum_{l=0}^{r-1} F_{k, l} y^{l}, \quad 1 \leq k \leq r
\end{gathered}
$$

where $Q, Q_{k} \in \mathbb{k}\left[x, y, z_{i, j}, a_{p}\right]$ and $G_{l}, F_{k, l} \in \mathbb{k}\left[x, z_{i, j}, a_{p}\right]$. Moreover, $\operatorname{deg}\left(f_{k}\left(z^{*}\right)\right) \leq d r$ and $\operatorname{deg}\left(\delta^{2}\left(z^{*}\right)\right) \leq 2 m(d-1) r$; hence, we get $\operatorname{deg}\left(F_{k, l}\right) \leq d r-l \leq 2 d s$ and $\operatorname{deg}\left(G_{l}\right) \leq$ $2 m(d-1) r-l \leq 4 m d s$ by the following lemma:

Lemma 4.10. Let $P(A, U, V) \in \mathbb{k}[A, U, V]$ where $U=\left(U_{1}, \ldots, U_{p}\right)$, $A=\left(A_{1}, \ldots, A_{r}\right)$ and $V$ is a single variable, and set $A(V):=V^{r}+A_{1} V^{r-1}+\cdots+$ $A_{r} \in \mathbb{k}[A, V]$. Let us consider the division of $P$ by $A$ with respect to $V: P=A Q+R$ with $\operatorname{deg}_{V}(R)<\operatorname{deg}_{V}(P)$. Then $\operatorname{deg}(R) \leq \operatorname{deg}(P)$.

Proof of Lemma 4.10. We can write $P(V):=P_{e} V^{e}+\cdots+P_{0}$ with $P_{i} \in \mathbb{k}[U], P_{e} \neq 0$ and $\operatorname{deg}\left(P_{i}\right) \leq d-i$ where $d:=\operatorname{deg}(P)$. Then we have:

$$
P=P_{e} V^{e-r} A(V)+R_{1}
$$

with

$$
R_{1}:=\left(P_{e-1}-P_{e} A_{1}\right) V^{e-1}+\cdots+\left(P_{e-r}-P_{e} A_{r}\right) V^{e-r}+P_{e-r-1} V^{e-r-1}+\cdots+P_{0}
$$

where $\operatorname{deg}_{V}\left(R_{1}\right)<\operatorname{deg}_{V}(P)$. Moreover, we see that $\operatorname{deg}\left(R_{1}\right) \leq d$. Thus, we obtain the result by induction on $e:=\operatorname{deg}_{V}(P)$.

Then, we have

$$
\begin{gathered}
\delta^{2}\left(\bar{z}^{*}\right)=\sum_{l=0}^{r-1} G_{l}\left(t, \bar{z}_{i, j}(x), \bar{a}_{p}(x)\right) y^{l} \bmod .(\bar{a}) \\
f_{k}\left(\bar{z}^{*}\right)=\sum_{l=0}^{r-1} F_{k, l}\left(t, \bar{z}_{i, j}(x), \bar{a}_{p}(x)\right) y^{l} \bmod .(\bar{a}), \quad 1 \leq k \leq r .
\end{gathered}
$$


However, $\delta^{2}\left(\bar{z}^{*}\right)=0$ mod. $(\bar{a})$ thus $G_{l}\left(x, \bar{z}_{i, j}(x), \bar{a}_{p}(x)\right)=0$ for all $l$. Moreover, $f_{k}(\bar{z})=$ 0 mod. $(\bar{a})+\mathfrak{m}^{\gamma}$, thus $f_{k}\left(\bar{z}^{*}\right)=0 \bmod .(\bar{a})+\mathfrak{m}^{\gamma}$ and $F_{k, l}\left(x, \bar{z}_{i, j}(x), \bar{a}_{p}(x)\right) \in(x)^{\gamma}$ for all $k$ and $l$ by Remark 6.6 [BM87].

By Theorem 4.2, there exist $\overline{\bar{z}}_{i, j}(x) \in \mathbb{k} \llbracket x \rrbracket$ and $\overline{\bar{a}}_{p}(x) \in \mathbb{k} \llbracket x \rrbracket$ for all $i, j$ and $p$, such that $G_{l}\left(x, \overline{\bar{z}}_{i, j}(x), \overline{\bar{a}}_{p}(x)\right)=0$ and $F_{k, l}\left(x, \overline{\bar{z}}_{i, j}(x), \overline{\bar{a}}_{p}(x)\right)=0$ for all $k$ and $l$ and $\bar{z}_{i, j}(x)-\overline{\bar{z}}_{i, j}(x), \bar{a}_{p}(x)-\overline{\bar{a}}_{p}(x) \in(x)^{c+2 s}$ for all $i, j$ and $p$.

Let us denote

$$
\begin{gathered}
\overline{\bar{a}}:=y^{r}+\overline{\bar{a}}_{1}(x) y^{r-1}+\cdots+\overline{\bar{a}}_{r}(x) \\
\overline{\bar{z}}_{i}:=\overline{\bar{a}} \bar{w}_{i}+\sum_{j=0}^{d-1} \overline{\bar{z}}_{i, j}(x) y^{j}
\end{gathered}
$$

for all $i$. It is straightforward to check that $f_{i}(\overline{\bar{z}})=0 \bmod . \delta^{2}(\overline{\bar{z}})$ for $1 \leq i \leq r$ and $\bar{z}_{j}(x)-\overline{\bar{z}}_{j}(x) \in \mathfrak{m}^{c+2 s}$ for $1 \leq j \leq m$.

Since ord $\left(\delta^{2}(\overline{\bar{z}})\right)=r$, then we have $f(\overline{\bar{z}}) \in \delta^{2}(\overline{\bar{z}}) \mathfrak{m}^{c+2 s-r}$. In any case, we have $f(\overline{\bar{z}}) \in \delta^{2}(\overline{\bar{z}}) \mathfrak{m}^{c}$. Then we use the following generalization of the Implicit Function Theorem:

Theorem 4.11 ([To72]). Let $\left(A, \mathfrak{m}_{A}\right)$ be a complete local ring and let $f_{1}(z), \ldots$, $f_{q}(z) \in A[z]$ with $q \leq m$. Let $\delta$ be a $q \times q$ minor of the Jacobian matrix $\frac{\partial\left(f_{1}, \ldots, f_{q}\right)}{\partial\left(z_{1}, \ldots, z_{m}\right)}$. Let us assume that there exists $\overline{\bar{z}}:=\left(\overline{\bar{z}}_{1}, \ldots, \overline{\bar{z}}_{m}\right) \in A^{m}$, such that

$$
f_{i}(\overline{\bar{z}}) \in(\delta(\overline{\bar{z}}))^{2} \mathfrak{m}_{A}^{c} \text { for all } 1 \leq i \leq q
$$

and for some $c \in \mathbb{N}$. Then there exists $\widetilde{z}=\left(\widetilde{z}_{1}, \ldots, \widetilde{z}_{m}\right) \in A^{m}$ such that

$$
f_{i}(\widetilde{z})=0 \text { for all } 1 \leq i \leq q \text {, and } \widetilde{z}_{j}-\overline{\bar{z}}_{j} \in(\delta(\bar{z})) \mathfrak{m}_{A}^{c} \text { for all } 1 \leq j \leq m .
$$

Thus, $\operatorname{ord}\left(k_{E}(\widetilde{z})\right)=\operatorname{ord}\left(k_{E}(\bar{z})\right)$ since $\widetilde{z}_{j}-\bar{z}_{j} \in \mathfrak{m}^{c+2 s-r}, 1 \leq j \leq m$, and since $\operatorname{ord}\left(k_{E}(\bar{z})\right)<s-\frac{r}{2} \leq 2 s-r$, hence $k_{E}(\widetilde{z}) \neq 0$. Since $k_{E}(z) f_{i}(z) \in\left(f_{1}, \ldots, f_{q}\right)$ for any $i$, we have $f_{i}(\widetilde{z})=0$ for $1 \leq i \leq n$.

Thus, we have proved that if $f(\bar{z}) \in \mathfrak{m}^{\gamma}$ and $H_{f_{1}, \ldots, f_{n}}(\bar{z}) \not \subset \mathfrak{m}^{s}$, then there exists $\widetilde{z} \in A^{m}$ such that $f(\widetilde{z})=0$ and $\widetilde{z}-\bar{z} \in \mathfrak{m}^{c}$. By Theorem 4.2, there exists $K$ such that $a(m, d) \leq d^{K^{m}}$ for all $d \geq 2, m \geq 1$. Since $c+2 s+1 \leq(c+1)(2 s+1)$ we have $\gamma \leq b(c+1)$ where

$$
b \leq(2 s+1)(4 m d s)^{K^{2(m+1) s}} \leq d^{K^{\prime m s}}, \quad \forall d \geq 2, \quad \forall m, s \geq 1,
$$

for some constant $K^{\prime}$ large enough (the existence of $K^{\prime}$ is proven exactly as the existence of $C$ in Remark 3.7). Thus, for any $d \geq 2, m \geq 1, f(z) \in A[z]^{n}$ such that $\operatorname{deg}(f) \leq d$ :

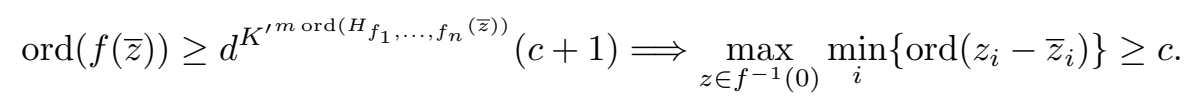

Hence, there exists $K>0$, such that

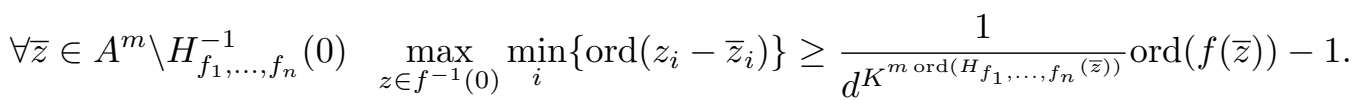

Thus

$$
\|f(\bar{z})\| \geq\left(\frac{1}{e} d\left(\bar{z}, f^{-1}(0)\right)\right)^{d^{K^{m o r d}\left(H_{f_{1}}, \ldots, f_{n}(\bar{z})\right)}}
$$


Since $K^{m \operatorname{ord}\left(H_{f_{1}, \ldots, f_{n}}(\bar{z})\right)}=\left\|H_{f_{1}, \ldots, f_{n}}(\bar{z})\right\|^{-\log (K) m}$, we have proved Inequality (4.1) with $K_{1}:=\frac{1}{e}$ and $K_{2}:=\log (K)$.

Let us prove the second inequality. Let $\bar{z} \in A^{m}$ such that $H(\bar{z}) \not \subset \mathfrak{m}^{s}$ and $I(\bar{z}) \subset \mathfrak{m}^{\gamma^{\prime}}$ where

$$
\gamma^{\prime}=a\left(2(m+1) s d^{C^{m}}, 4 m d s d^{C^{m}}\right)\left(c+2 s d^{C^{m}}+1\right),
$$

where $C$ is the constant of Remark 3.7, i.e., $\gamma^{\prime}=\gamma\left(m, d, s d^{C^{m}}, c\right)$. In particular, $\sqrt{H+I}(\bar{z}) \not \subset \mathfrak{m}^{s}$. Then we have $\sqrt{H+I}^{d^{C^{m}}}(\bar{z}) \not \subset \mathfrak{m}^{s d^{C^{m}}}$, thus $\left(H_{f_{1}, \ldots, f_{n}}+I\right)(\bar{z}) \not \subset$ $\mathfrak{m}^{s d^{C^{m}}}$ since $\sqrt{H+I} d^{C^{m}} \subset H_{f_{1}, \ldots, f_{n}}+I$ by Corollary 3.5 and Remark 3.7. However, $I(\bar{z}) \subset \mathfrak{m}^{\gamma^{\prime}} \subset \mathfrak{m}^{s d^{C^{m}}}$, then $H_{f_{1}, \ldots, f_{n}}(\bar{z}) \not \subset \mathfrak{m}^{s d^{C^{m}}}$. Thus, by the previous case (by replacing $s$ by $s d^{C^{m}}$ ), we see that there exists $\widetilde{z} \in A^{m}$ such that $f(\widetilde{z})=0$ and $\widetilde{z}-\bar{z} \in \mathfrak{m}^{c}$. Moreover, there exists a constant $K^{\prime \prime}>0$ such that $d^{C^{m}} m \leq d^{K^{\prime \prime m}}$ for all $d \geq 2$ and all $m \geq 1$; thus, we have

$$
\gamma^{\prime} \leq d^{K^{\prime m s d} C^{m}} \leq d^{K^{\prime s d} K^{\prime \prime m}}
$$

Hence, exactly as the end of the proof of Inequality (4.1), we have

$$
\|f(\bar{z})\| \geq\left(K_{1} d\left(\bar{z}, f^{-1}(0)\right)\right)^{d}\left(\frac{1}{\|H(\bar{z})\|}\right)^{d^{K_{3}^{m}}}
$$

for some positive constants $K_{1}>0$ and $K_{3}>0$ independent of $\bar{z}$.

Example 4.12. Let $f(z) \in \mathbb{k}[z]^{n}$ and let us assume that $z_{m} \in \sqrt{H_{f}}$. Let $h \in$ $(x, y)^{2} \mathbb{k} \llbracket x, y \rrbracket$ be a non-zero power series without multiple factor and let

$$
g\left(z_{1}, \ldots, z_{m-1}\right):=f\left(z_{1}, \ldots, z_{m-1}, h\right) .
$$

Then, we claim that the Artin function of $g$ is bounded by an affine function.

Indeed, let us denote by $e$ the order of $h$. By Theorem 4.4 there exist two constants $a \geq 0$ and $b \geq 0$ (depending on $e$ and a power of $z_{m}$ which belongs to $H_{f}$ ) such that for any $\bar{z}_{1}, \ldots, \bar{z}_{m-1} \in \mathbb{k} \llbracket x, y \rrbracket$ with

$$
f\left(\bar{z}_{1}, \ldots, \bar{z}_{m-1}, h\right) \in(x, y)^{a c+b}
$$

there exists $\widetilde{z}_{1}, \ldots, \widetilde{z}_{m-1}, \widetilde{h} \in \mathbb{k} \llbracket x, y \rrbracket$ such that

$$
\begin{gathered}
f\left(\widetilde{z}_{1}, \ldots, \widetilde{z}_{m-1}, \widetilde{h}\right)=0 \\
\text { and } \widetilde{z}_{i}-\bar{z}_{i}, \widetilde{h}-h \in(x, y)^{c}, \quad \forall i .
\end{gathered}
$$

We claim that $J:=\left(\frac{\partial h}{\partial x}, \frac{\partial h}{\partial y}\right)$, the Jacobian ideal of $h$, contains a power of $(x, y)$. Indeed, it is well known that $h \in \sqrt{J}$ (see Theorem 7.1.5 [HS], for example) and after a linear change of coordinates we can write $h=g . u$ where $g$ is a Weierstrass polynomial in the variable $y$ and $u$ is a unit. Since $u$ is a unit and $J$ does not depend on the choice of coordinates, we have $\sqrt{J}=\sqrt{J^{\prime}}$ where $J^{\prime}$ is the Jacobian ideal of $g$. Moreover $g$ and $\frac{\partial g}{\partial y}$ are coprime since $g$ is a polynomial with no multiple factor and $\operatorname{char}(\mathbb{k})=0$. However, $g \in \sqrt{J^{\prime}}$ thus height $\left(\sqrt{J^{\prime}}\right)=2$ and $\sqrt{J}=\sqrt{J^{\prime}}=(x, y)$ since $(x, y)$ is the only height two radical ideal of $\mathbb{k} \llbracket x, y \rrbracket$. This proves that $J$ contains a power of $(x, y)$. 
Thus there exists $k \in \mathbb{N}$ such that $(x, y)^{k} \subset J^{2}$. Let us assume that $c>k+1$ and let us consider the equation:

$$
P\left(x, y, x_{1}, y_{1}\right):=\widetilde{h}(x, y)-h\left(x_{1}, y_{1}\right)=0
$$

where $x_{1}$ and $y_{1}$ are new variables. Then

$$
\begin{aligned}
P(x, y, x, y) \in(x, y)^{c} \subset(x, y)^{c-k} & \left(\frac{\partial h}{\partial x}(x, y), \frac{\partial h}{\partial y}(x, y)\right)^{2} \\
= & (x, y)^{c-k}\left(\frac{\partial P}{\partial x_{1}}(x, y, x, y), \frac{\partial P}{\partial y_{1}}(x, y, x, y)\right)^{2}
\end{aligned}
$$

since $\frac{\partial P}{\partial x_{1}}(x, y, x, y)=-\frac{\partial h}{\partial x}(x, y)$ and $\frac{\partial P}{\partial y_{1}}(x, y, x, y)=-\frac{\partial h}{\partial y}(x, y)$. Thus by Theorem 4.11, there exists $\bar{x}_{1}, \bar{y}_{1} \in \mathbb{k} \llbracket x, y \rrbracket$ such that $P\left(x, y, \bar{x}_{1}, \bar{y}_{1}\right)=0$ and $\bar{x}_{1}(x, y)-x$, $\bar{y}_{1}(x, y)-y \in(x, y)^{c-k}$. Thus, the $\mathbb{k}$-morphism $\varphi$ defined by $\varphi(p(x, y)):=p\left(\bar{x}_{1}(x, y), \bar{y}_{1}\right.$ $(x, y))$, for all $p \in \mathbb{k} \llbracket x, y \rrbracket$, is a $\mathbb{k}$-automorphism of $\mathbb{k} \llbracket x, y \rrbracket$. By assumption $\varphi(h)=\widetilde{h}$ and $\varphi(p)-p \in(x, y)^{c-k}$ for any $p \in \mathbb{k} \llbracket x, y \rrbracket$. Let $\widetilde{z}_{i}^{\prime}:=\varphi^{-1}\left(\widetilde{z}_{i}\right)$ for $1 \leq i \leq m$. Then $\widetilde{z}_{i}^{\prime}-z_{i} \in(x, y)^{c-k}$, for $1 \leq i \leq m$, and $f\left(\widetilde{z}_{1}^{\prime}, \ldots, \widetilde{z}_{m}^{\prime}, h\right)=0$. This proves that the Artin function of $g$ is bounded by $c \longmapsto a(c+k)+b$.

From Theorem 4.4 we can find the following bound of the Artin function of an isolated singularity (a similar bound has been given in [Ro10] for binomial ideals):

Corollary 4.13. Let $\mathbb{k}$ be a perfect field. Let $I=\left(f_{1}, \ldots, f_{n}\right)$ be an ideal of $\mathbb{k}\left[x, y, z_{1}\right.$, $\left.\ldots, z_{m}\right]$ such that $I \subset\left(z_{1}, \ldots, z_{m}\right)$. Let us assume that $H_{f_{1}, \ldots, f_{n}}$ contains a power of the ideal $\left(z_{1}, \ldots, z_{m}\right)$. Then the Artin function of $I$ is bounded by a function of the form $c \longmapsto K^{K^{c}}$ for some constant $K>0$.

Proof. Let $A$ denote the $\operatorname{ring} \mathbb{k} \llbracket x, y \rrbracket$. For $\bar{z} \in A^{m}$ let us set $D(\bar{z}):=\min _{i} \operatorname{ord}\left(\bar{z}_{i}\right)$. Let $k \in \mathbb{N}$ such that $(z)^{k} \subset H_{f_{1}, \ldots, f_{m}}$ and let $d \in \mathbb{N}$ be a bound of the degrees of the $f_{i}$ 's.

By Theorem 4.4, for any $\bar{z} \in A^{m}$ such that $D(\bar{z})<\infty$, if $I(\bar{z}) \in \mathfrak{m}^{d^{K_{1}^{m k(D(\bar{z})+1)}}}(c+1)$, then there exists $\widetilde{z} \in A^{m}$ such that $I(\widetilde{z})=0$ and $\widetilde{z}-\bar{z} \in \mathfrak{m}^{c} A^{m}$ (see Inequality (4.3) in the proof of Theorem 4.4). Let us remark that there exists a constant $K>1$ such that $d^{K_{1}^{m k c}}(c+1) \leq K^{K^{c}}$ for any $c \geq 1$ (this inequality is proven exactly as the existence of $C$ in Remark 3.7).

Now let $\bar{z}$ be any element of $A^{m}$ such that $I(\bar{z}) \in \mathfrak{m}^{K^{K^{c}}}$. Then two cases may occur: either $D(\bar{z}) \geq c$, either $D(\bar{z})<c$. In the first case, let us set $\widetilde{z}_{j}:=0$ for $1 \leq j \leq m$. If $D(\bar{z})<c$, then $I(\bar{z}) \in \mathfrak{m}^{d^{K_{1}^{m k(D(\bar{z})+1)}}(c+1)}$ by assumption on $K$, thus there exists $\widetilde{z} \in A^{m}$ such that $I(\widetilde{z})=0$ and $\widetilde{z}_{j}-\bar{z}_{j} \in \mathfrak{m}^{c}$ for $1 \leq j \leq m$. This proves the corollary.

\section{Acknowledgments}

I would like to thank Michel Hickel for his comments about a previous version of this paper. I also thank the referee for its relevant comments and remarks that helped to improve the presentation. 


\section{References}

[Ar69] M. Artin, Algebraic approximation of structures over complete local rings, Publ. Math. IHES, 36 (1969), 23-58.

[Ar70] M. Artin, Construction techniques for algebraic spaces, Actes Congres Int. Math., 1 (1970), 419-423.

[BM87] E. Bierstone and P. Milman, Relations among analytic functions I, Ann. Inst. Fourier, 37(1) (1987), 187-239.

[El73] R. Elkik, Solutions d'équations à coefficients dans un anneau hensélien, Ann. Sci. École Norm. Sup. (4), 6 (1973), 553-604.

[G-R03] O. Gabber and L. Ramero, Almost ring theory, Lecture Notes in Mathematics, 1800, Springer-Verlag, Berlin, 2003.

[Gr66] M.J. Greenberg, Rational points in Henselian discrete valuation rings, Publ. Math. IHES, 31, (1966), 59-64.

[HS] C. Huneke and I. Swanson, Integral closure of ideals, rings, and modules, London Mathematical Society Lecture Note Series, 336. Cambridge University Press, Cambridge, 2006.

[Ł59] S. Łojasiewicz, Sur le problème de la division, Studia Math., 18, (1959), 87-136.

[Po86] D. Popescu, General Néron desingularization and approximation, Nagoya Math. J., 104, (1986), 85-115.

[Po00] D. Popescu, Artin approximation, Handbook of Algebra, 2, 321-356, North-Holland, Amsterdam, 2000.

[Ro05] G. Rond, Sur la linéarité de la fonction de Artin, Ann. Sci. École Norm. Sup. (4), 38(6) (2005), 979-988.

[Ro06] G. Rond, Lemme d'Artin-Rees, théorème d'Izumi et fonctions de Artin, J. Algebra, 299(1), (2006), 245-275.

[Ro10] G. Rond, Bornes effectives des fonctions d'approximation des solutions formelles d'équations binomiales, J. Algebra, 323(9) (2010), 2547-2555.

[Se74] A. Seidenberg, Constructions in Algebra, Trans. AMS, 197 (1974), 273-313.

[Sp99] M. Spivakovsky, A new proof of D. Popescu's theorem on smoothing of ring homomorphisms, J. Amer. Math. Soc., 12(2) (1999), 381-444.

[Te90] B. Teissier, Résultats récents d'algèbre commutative effective, Séminaire Bourbaki, Vol. 1989/90, Astérisque, No. 189-190, (1990), Exp. No. 718, 107-131.

[Te12] B. Teissier, Some resonances of Eojasiewicz inequalities, Wiadomości Matematyczne, 48(2), 2012, 271-284.

[To72] J.-C. Tougeron, Idéaux de fonctions différentiables, Ergebnisse der Mathematik und ihrer Grenzgebiete, Band 71. Springer-Verlag, Berlin, New York, (1972).

Institut de Mathématiques de Luminy, Faculté des Sciences de Luminy, Case 907, 163 AV. De Luminy, 13288 Marseille Cedex 9, France

E-mail address: rond@iml.univ-mrs.fr 
